\title{
Analyzing coefficients of psychophysical power functions
}

\author{
STANLEY J. RULE \\ University of Alberta, Edmonton, Alberta, Canada
}

\begin{abstract}
Some mathematical properties of coefficients of power functions were analyzed. The size of correlations between intercepts (the logarithm of the coefficient) and exponents depends on the choice of unit of measurement of the physical stimuli. When the mean of logarithms of a set of responses is uncorrelated with the exponent, the absolute size of the correlation between the intercept and the exponent increases as the geometric mean of the stimulus measures deviates from one. When the geometric mean is less than one, the correlation is positive, and when it is greater than one, the correlation is negative. Similar trends hold for a nonzero correlation between the exponent and the mean logarithm of a set of responses. The power of statistical tests of differences between mean intercepts also depends on the geometric mean of the stimuli. Power is reduced as the geometric mean deviates from one. Effects are illustrated with real data.
\end{abstract}

When psychophysical power functions are fitted to data from magnitude estimation, the primary emphasis in the analysis is usually given to estimates of the exponent. The present paper, however, focuses on the coefficient, the other parameter of the two-parameter power function. The coefficient is a dimensional constant, whose value depends on the unit of measurement for the subjects' responses and for the physical stimuli. The coefficient has been interpreted as indicating the size of numbers that subjects use in making their magnitude estimates, provided that the scale for measuring stimuli remains constant (e.g., Gescheider, 1988). Borg and Marks (1983) have listed 12 factors that can affect the value of the coefficient by influencing either the size of the response or the measure of the stimulus.

A number of researchers have examined the relation between the coefficient (or its logarithm) and the exponent for different experimental conditions or for individual subjects (e.g., Baird, Kreindler, \& Jones, 1971; Gescheider \& Hughson, 1991; Kowal, 1987; Robinson, 1992; Stevens, 1974), and most have found the coefficient to be negatively related to the exponent. From the point of view of the coefficient as an indication of the size of the response, the negative relation would suggest that subjects or experimental conditions that produce greater responses tend to yield lower exponents.

Researchers have also subjected differences in average coefficients (or their logarithms) from groups of subjects to tests of significance (e.g., Algom, Marks, \& Wiesenfeld, 1991; Butler \& Overshiner, 1983; Ferguson \& Mar-

This research was supported by Grant AO151 from the Natural Sciences and Engineering Research Council of Canada. The author wishes to thank Charles M. Bourassa for his critical comments on this manuscript. Correspondence should be sent to S. J. Rule, Department of Psychology, University of Alberta, Edmonton, AB, Canada, T6G 2E9. tin, 1983; Gescheider \& Hughson, 1991; Kowal, 1987; Robinson, 1992). Some of these studies were designed to show that the unit of subjects' responses is stable with respect to changes in experimental procedure or with the same experimental procedure on different occasions. In these studies, nonsignificant outcomes have been interpreted as indicating similar units for the sets of responses.

The present paper examines some pitfalls in basing conclusions on analyses of coefficients. Its focus is on interpreting correlations between coefficients and other variables, and on tests of significance of the difference between average coefficients. In particular, it will be shown that interpreting the coefficient as reflecting the size of a set of responses can be misleading, that a negative correlation between the coefficient and exponent is not psychologically meaningful, and that conclusions concerning similar units of response drawn from nonsignificant differences between mean coefficients are questionable.

To motivate the developments that follow, consider two analyses of data, which will be presented in greater detail later. The first analysis examines the relation between the coefficient and exponent from individual subjects' data. The data are magnitude estimates of the area of a set of circles ranging in physical area from $12.5 \mathrm{~cm}^{2}$ to $239.2 \mathrm{~cm}^{2}$. When power functions were fitted to each subject's data, the logarithms of the coefficients were found to be negatively related to the exponents, with a correlation coefficient of nearly -1.0 . Negative correlations have been reported before, as I have noted above. The analysis was repeated, but for the reanalysis the area of the circles was measured in square meters rather than square centimeters, and, consequently, they ranged from $.00125 \mathrm{~m}^{2}$ to $.02392 \mathrm{~m}^{2}$. Power functions were again fitted to each subject's magnitude estimates. This time, however, the opposite results were found. The logarithms of coefficients were positively related to the subjects' exponents, and the correlation coefficient was nearly $+\mathbf{1 . 0}$. 
In a second example, which is concerned with significance tests between average coefficients, two groups of subjects gave magnitude estimates of areas of circles, which also ranged in area from $12.5 \mathrm{~cm}^{2}$ to $239.2 \mathrm{~cm}^{2}$. A test between the means of the logarithms of coefficients was far from significant, despite the fact that all responses in one group had been artificially increased by $50 \%$. It would appear that the test lacked sufficient power to detect a difference of this size. However, when the analysis was repeated with the area of circles measured in square inches rather than square centimeters, the outcome was highly significant.

As the two preceding examples illustrate, there are problems in interpreting analyses of coefficients of power functions. However, such results are to be expected from the mathematical properties of the coefficient. The purpose of the developments that follow is to show what relations among coefficients, exponents, and average responses are to be expected solely on the bases of mathematical considerations in the absence of any experimental effect. This is not to imply that experimental effects do not occur. But when they do, they may be masked by relations that are due to the properties of the measures.

\section{PROPERTIES OF THE COEFFICIENT (INTERCEPT)}

Consider a power function fitted to magnitude estimates from subject $i$ :

$$
V_{i j}=c_{i} S_{j}^{b_{i}}
$$

where $V_{i j}$ denotes the $i$ th subject's magnitude estimate of the $j$ th stimulus, $S_{j}$ is the physical measure of the $j$ th stimulus, and $c_{i}$ and $b_{i}$ are the coefficient and exponent, respectively. In Equation 1 and in the following developments, the error component in fitting power functions to subjects' data has been ignored. How well the functions fit the data does not affect the conclusions, and ignoring goodness of fit as well as distinctions between parameters and their estimates helped to simplify notation.

Taking logarithms of both sides of Equation 1 yields the familiar linear form that is usually fitted to the data by a linear least squares solution:

$$
\log V_{i j}=b_{i} \log S_{j}+\log c_{i}
$$

Let $Y_{i j}=\log V_{i j}, X_{j}=\log S_{j}$, and $a_{i}=\log c_{i}$; Equation 2 may then be expressed in simpler form as

$$
Y_{i j}=b_{i} X_{j}+a_{i}
$$

Because the logarithm of the power function's coefficient is also the intercept in Equation 3, it has become common practice to refer to it as the intercept, a practice that is followed in the present paper. For a least squares solution, the intercept is computed by

$$
a_{i}=\bar{Y}_{i}-b_{i} \bar{X}
$$

where $\bar{Y}_{i}$ is the average response given by subject $i$ to the set of stimuli, and $\bar{X}$ is the average stimulus value (after logarithmic transformations).

To determine the expected relations among the variables in Equation 4 in the absence of experimental effects, it is assumed that subjects vary in their average response to the set of stimuli and in values of their exponents and intercepts, but that there are no other experimental relations. In particular, it is assumed that the values of $\bar{Y}_{i}$ are uncorrelated with exponents $b_{i}$. The case for which $\bar{Y}_{i}$ is correlated with $b_{i}$ is considered later. For this analysis, it is assumed that

$$
r_{\bar{Y} b}=0 .
$$

Because the intercept is computed by a linear combination of $b_{i}$ and $\bar{Y}_{i}$ as shown in Equation 4, the multiple correlation $R_{a, \bar{Y} b}$ for predicting the intercept $a_{i}$ from $\bar{Y}_{i}$ and $b_{i}$ is necessarily 1.0 . And from the assumption that the exponent does not depend on the average response, expressed as Equation 5, the squared multiple correlation is equal to

$$
R_{a . \bar{Y} b}^{2}=r_{a \bar{Y}}^{2}+r_{a b}^{2}=1.0 .
$$

Equation 6 indicates a trading relation between the squared correlation of average responses with intercepts and the squared correlation of exponents with intercepts such that

$$
r_{a b}^{2}=1-r_{a}^{2} \bar{Y} .
$$

According to Equation 7, the squared correlation between the intercept and exponent is low when the squared correlation between the intercept and the average size of the numerical responses is high and, conversely, the squared correlation between the intercept and exponent is high when the relation between the intercept and the average size of the response is weak. These relations are opposite to what would be required for a response unit interpretation of relations between intercepts and exponents. Such an interpretation requires a high correlation between the intercept and the average size of numerical responses when intercepts correlate with exponents, contrary to Equation 7.

A consequence of Equation 7 that seems particularly contradictory for an interpretation of the intercept as a measure of the unit of subjects' responses is seen with modulus equalization. Modulus equalization is an analytic procedure that is sometimes used to equalize subjects' units of measurement (Engen, 1971). In the procedure, each subject's responses are rescaled so that every subject's data have the same geometric mean. Consequently, after modulus equalization, the correlation between the intercept and the constant average response is zero, whereas the squared correlation between the intercept and the exponent is one. That is, there is a perfect relationship between the intercept and the exponent when the intercept is unrelated to the unit of subjects' magnitude judgments. 
What these examples show is that an interpretation of the intercept as reflecting the unit of measurement of subjects' magnitude estimates can produce misleading conclusions. The problem is that the relation between the response unit and the intercept is one directional. An increase in the size of responses yields an increase in the intercept, but an increase in the intercept does not necessarily stem from an increase in the size of responses. There are other factors that determine the size of the intercept. One factor that influences the size of the intercept and its relations to other variables is the physical measure of the stimuli. Unfortunately, the physical stimulus enters into the relation in a way that leaves analyses of intercepts open to misinterpretation, because the stimulus measure not only affects the value of the intercept, it also influences the nature of relations between the intercept and other variables.

\section{Influence of Measures of the Stimulus}

To help explicate the influence of physical measures on relations among the variables, correlations and their squares are expressed in terms of variances. For purposes of simplifying notation, it is assumed in the following developments that the number of subjects is sufficiently great that no distinction needs to be made between population and sample values of variances and correlation coefficients. This assumption does not alter any conclusion drawn from the developments.

From the formula for computing $a_{i}$, presented as Equation 4 , and from the assumption of zero correlation between intercepts and exponents, presented as Equation 5, the correlation between the exponent and the intercept may be expressed as

$$
r_{a b}=\frac{-\bar{X} \sigma_{b}}{\left(\sigma_{\bar{Y}}^{2}+\bar{X}^{2} \sigma_{b}^{2}\right)^{1 / 2}},
$$

and the correlation between the mean logarithm of each subject's responses and intercepts may be expressed as

$$
r_{a} \bar{Y}=\frac{\sigma_{\bar{Y}}}{\left(\sigma_{\bar{Y}}^{2}+\bar{X}^{2} \sigma_{b}^{2}\right)^{1 / 2}} .
$$

Squaring both sides of Equations 8 and 9 yields

$$
r_{a b}^{2}=\frac{\bar{X}^{2} \sigma_{b}^{2}}{\sigma_{\bar{Y}}^{2}+\bar{X}^{2} \sigma_{b}^{2}},
$$

and

$$
r_{a \bar{Y}}^{2}=\frac{\sigma_{\bar{Y}}^{2}}{\sigma_{\bar{Y}}^{2}+\bar{X}^{2} \sigma_{b}^{2}} .
$$

Equations 10 and 11 for squared correlations show that an increase in the square of the mean logarithm of the stimuli increases the squared correlation between the intercept and exponent and decreases the squared correlation between the intercept and $\bar{Y}_{i}$.

The expressions for the unsquared correlations presented as Equations 8 and 9 reveal a major problem in interpreting analyses of intercepts-namely, that the results are influenced by the choice of measurement scale for the stimuli. Consider a change of unit for the physical measures such that the measure of the $j$ th stimulus on the new scale is now $S_{j}^{\prime}$, which is related to the original measure by

$$
S_{j}^{\prime}=g S_{j},
$$

and, hence,

$$
\begin{aligned}
X_{j}^{\prime} & =\log S_{j}^{\prime} \\
& =\log g+\log S_{j} \\
& =d+X_{j},
\end{aligned}
$$

where $d=\log g$. A change in scale for the physical stimulus affects neither the subjects' responses nor their exponents, but it does influence the intercept such that

$$
\begin{aligned}
a_{i}^{\prime} & =\bar{Y}_{i}-b_{i}(d+\bar{X}) \\
& =a_{i}-b_{i} d .
\end{aligned}
$$

A change of scale also affects correlations with the intercept. The correlation coefficients given in Equations 8 and 9 for the new intercept $a_{i}^{\prime}$ are

$$
r_{a^{\prime} b}=\frac{-(d+\bar{X}) \sigma_{b}}{\left[\sigma_{\bar{Y}}^{2}+(d+\bar{X})^{2} \sigma_{b}^{2}\right]^{1 / 2}},
$$

and

$$
r_{a^{\prime} \bar{Y}}=\frac{\sigma_{\bar{Y}}}{\left[\sigma_{\bar{Y}}^{2}+(d+\bar{X})^{2} \sigma_{b}^{2}\right]^{1 / 2}} .
$$

Equations 14 and 15 are graphed in Figure 1, which presents $r_{a^{\prime} b}$ and $r_{a^{\prime}} \bar{Y}$ as functions of $\bar{X}^{\prime}=\bar{X}+d$ for three different ratios of the standard deviations $\sigma_{\bar{Y}} / \sigma_{b}$. The trends are similar in the three cases. The relative values of the standard deviations affect only the steepness of the curves.

According to Equation 14, and as illustrated in Figure 1 , the correlation $r_{a^{\prime} b}$ between the intercept and the exponent can be positive or negative, depending on the sign of $\bar{X}^{\prime}=\bar{X}+d$. When $d$ is a large positive value such that $\bar{X}^{\prime}$ is positive, the correlation is negative, and, conversely, when $d$ is a large negative value such that $\bar{X}^{\prime}$ is negative, the correlation is positive. When $\bar{X}^{\prime}$ is zero, the correlation is zero, and as $\bar{X}^{\prime}$ deviates from zero, the absolute value of $r_{a^{\prime} b}$ increases. With respect to the untransformed stimulus measures (i.e., not logarithmically transformed), negative and positive values of $\bar{X}^{\prime}$ correspond to geometric means of stimulus measures $S_{j}^{\prime}$ that are less than or greater than one. When the geometric mean is greater than one, the correlation is negative, and when it is less than one, the correlation is positive. For a geometric mean of one, the correlation is zero. For most studies, a unit of measurement is selected such that the geometric mean of the stimuli is greater than one. Hence, a negative correlation between the intercept and the exponent is to be expected. 

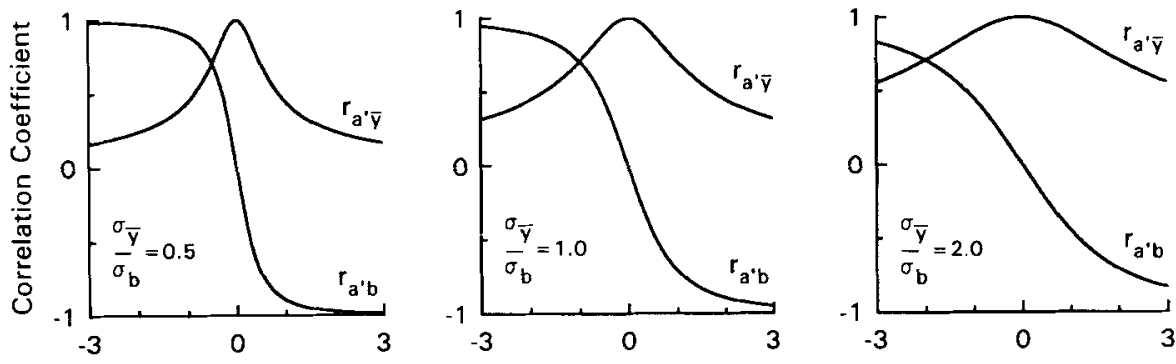

Mean Logarithm of Stimulus Measure $\left(\bar{X}^{\prime}\right)$

Figure 1. Correlation between the intercept and exponent $\left(r_{a^{\prime}} b\right)$ and correlation between the intercept and mean logarithm of responses $\left(r_{a}, \bar{Y}\right)$ as a function of the mean logarithm of the stimulus measure $\left(\bar{X}^{\prime}\right)$, for three ratios of standard deviations and for zero correlation between the mean logarithm of responses and the exponent.

As shown in Figure 1, the trend for the correlation $r_{a}, \bar{Y}$ between the intercept and $\bar{Y}_{i}$ is opposite to that for the absolute value of $r_{a^{\prime} b}$. When $\bar{X}^{\prime}$ is zero, $r_{a^{\prime}} \bar{Y}$ is one, and as $\bar{X}^{\prime}$ deviates from zero, $r_{a^{\prime}} \bar{Y}$ decreases.

In the developments above, it has been assumed that there are no experimental effects within the data. Specifically, it has been assumed that $r_{\bar{Y} b}$ is zero. The question arises, what are the influences of the physical scale for measuring the stimuli when the size of the response is correlated with the exponent?

\section{Influence of Correlations Between \\ Responses and Exponents}

When $r_{\bar{Y} b}$ is nonzero, it enters into equations for $r_{a^{\prime} b}$ and $r_{a^{\prime}} \bar{Y}$, making the expressions more complicated. ${ }^{1}$ However, the general trends remain. As $\bar{X}^{\prime}$ increases, $r_{a^{\prime}} b$ decreases, while $r_{a^{\prime}} \bar{Y}$ increases to unity and then decreases. These trends are illustrated in Figure 2 for $r_{\bar{Y} b}=$ -.5 and $r_{\bar{Y} b}=.5$. The ratio of standard deviations $\sigma_{\bar{Y}} / \sigma_{b}$ is set to one in the illustrations. In general, when $\bar{X}^{\prime}=0.0, r_{a^{\prime}} \bar{Y}=1.0$, and $r_{a^{\prime} b}=r_{\bar{Y} b}$, and when $\bar{X}^{\prime}=$ $r_{\bar{Y} b} \sigma_{\bar{Y}} / \sigma_{b}, r_{a^{\prime} b}=0$.

A nonzero correlation $r_{\bar{Y} b}$ is present when an experimental condition affects both the exponent and the size of the response. Some of these cases have produced sets

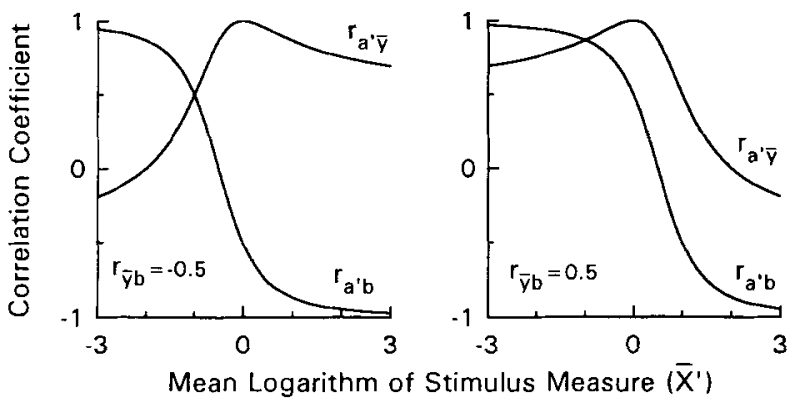

Figure 2. Correlation between the intercept and exponent $\left(r_{a^{\prime}} b\right)$ and correlation between the intercept and mean logarithm of responses $\left(r_{a}, \bar{Y}\right)$ as a function of the mean logarithm of the stimulus measure $\left(\bar{X}^{\prime}\right)$, for two correlations between mean logarithm of responses and the exponent $\left(r_{\bar{Y} b}\right)$. of power functions that appear to converge, a result that has been particularly relevant to findings of a negative relation between exponents and intercepts. Stevens (1974) has summarized experiments yielding converging power functions for a variety of stimulus conditions. In these cases, the subjects judge the magnitude of one stimulus dimension for different fixed values of a second stimulus dimension. For example, a set of converging power functions has been found to describe the size-weight illusion (Cross \& Rotkin, 1975; Rule \& Curtis, 1977; Stevens \& Rubin, 1970). Each function describes the relation between magnitude estimation of heaviness and physical weight for a different-sized object. Heaviness judgments decrease and exponents increase as the size of the objects become larger and, consequently, the correlation between average responses and exponents $r_{\bar{Y} b}$ is negative.

Stevens and Rubin (1970) have shown that when power functions converge to a point, the exponents of the functions are linearly related to the intercepts, and the coordinates of the convergence point can be estimated from the linear relation. Typically, the relation between intercepts and exponents has been reported as negative. However, the direction of the relation is of no theoretical consequence. As the graphs in Figure 2 illustrate, its direction is an artifact of the measurement scale of the stimuli. A negative relation is found because experimenters usually select a measurement scale that yields larger, more convenient, values. It can be seen from the left panel in Figure 2 that a positive relation between intercepts and exponents requires stimulus measures that are less than one, sometimes much less. Such small, fractional values are simply less convenient (cf. $50 \mathrm{~g}$ and $0.05 \mathrm{~kg}$ ).

The trends in Figures 1 and 2 show how the scale for measuring the stimuli can be selected to set stimulus measures sufficiently low or sufficiently high to yield almost perfect positive or negative correlations, regardless of what experimental effects are present in the data.

\section{Illustrative Data}

To illustrate the developments above, a set of real data was analyzed. The data are magnitude estimates of circle area from Rule and Markley (1971). The stimuli were 
seven outline circles, which varied in area from $12.5 \mathrm{~cm}^{2}$ to $239.2 \mathrm{~cm}^{2}$ in equal logarithmic steps. The middle stimulus was presented first as a standard and was assigned the modulus 10. The data were obtained from 48 subjects.

The geometric mean of all the subjects' responses is 10.05 , which is in line with the assignment of the modulus 10 to a standard at the geometric mean of the set of stimuli. Of primary interest are the correlations among estimates of parameters of the power function. The correlation between intercepts and exponents for individual subjects is -.94 , and the correlation between the intercept and $\bar{Y}_{i}$ is .31 . If the intercept were interpreted as indicating subjects' units of measurement, the negative correlation between the intercept and the exponent would lead to the erroneous conclusion that subjects' exponents decreased as the size of their responses increased. However, the correlation between the mean logarithm of each subject's responses $\bar{Y}_{i}$ and the exponent is only .05 , which indicates little or no relation between size of response and the exponent. Instead, the size of $r_{a^{\prime} b}$ is a consequence of the logarithm of the geometric mean of the stimuli, $54.5 \mathrm{~cm}^{2}$, entering into the relation.

To illustrate the influence of the unit of measurement of the stimuli on the correlations, the data were reanalyzed with area measured in square millimeters, which provides a geometric mean of stimulus measures of $5,450 \mathrm{~mm}^{2}$. As expected, increasing the geometric mean produced a correlation between intercepts and exponents that increased in absolute value, $r_{a^{\prime} b}=-.99$, and a correlation between intercepts and average responses that decreased in value, $r_{a^{\prime}} \bar{Y}=.12$. An analysis with area measured in square meters, which provides a stimulus geometric mean of $0.00545 \mathrm{~m}^{2}$, yielded a positive correlation for $r_{a^{\prime} b}$ of .96 . A positive value is expected from the low value of the geometric mean of the stimulus measures whose logarithm is negative. The value of $r_{a^{\prime}} \bar{Y}$ for this analysis is .32 .

Figure 3 presents a geometric illustration of how a change in the unit of measurement of the stimuli influences the relation between intercepts and exponents. In the figure, lines representing three hypothetical power functions with different exponents for perceived area are plotted on logarithmic coordinates. Because the exponents (slopes) vary, all pairs of lines intersect. The two vertical axes labeled $\mathrm{cm}^{2}$ and $\mathrm{m}^{2}$ pass through the respective origins for area measured in either square centimeters or square meters. Because the origin on a logarithmic scale corresponds to unity on the untransformed scale, a change in the unit of the physical measure corresponds to a lateral shift of the vertical axis. Figure 3 illustrates how an axis to the left of the region within which all pairs of lines intersect (e.g., the axis for square centimeters) yields intercepts that are negatively related to exponents, and an axis to the right of the region of intersection (e.g., the axis for square meters) yields intercepts that are positively related to exponents. If the stimuli were rescaled to a unit of measurement such that the axis passed through the region of intersection, the result would be a relation between intercepts and exponents that is closer to zero.

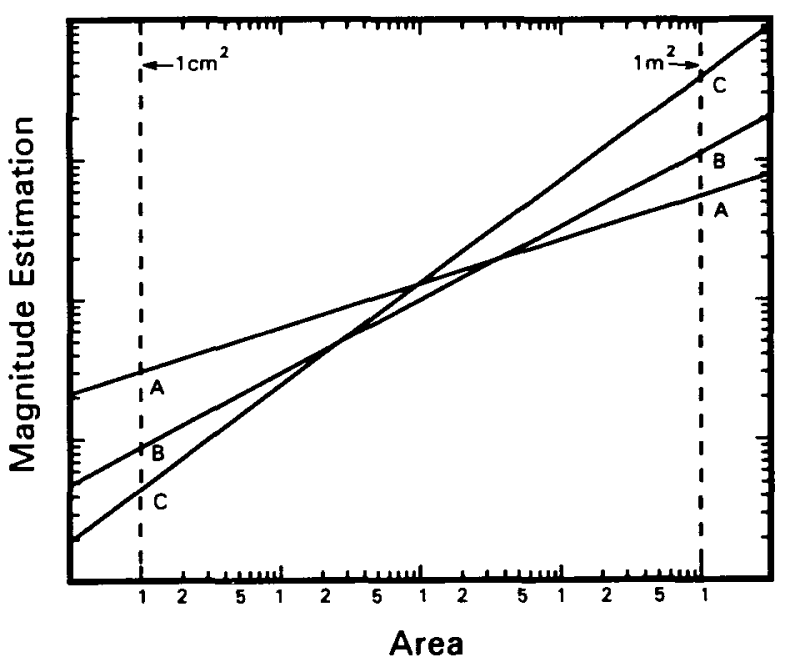

Figure 3. Hypothetical curves for magnitude estimation of perceived area plotted on logarithmic coordinates with scale units omitted. Vertical lines labeled $\mathrm{cm}^{2}$ and $\mathrm{m}^{2}$ represent axes through origins for area measured in square centimeters and square meters, respectively.

Clearly, as the preceding analyses illustrate, the absolute value and direction of $r_{a^{\prime}} b$ as well as the value of $r_{a^{\prime}} \vec{Y}$ depend upon the arbitrary choice of a measurement unit for the physical stimulus. As such, they provide no information of scientific importance.

\section{SIGNIFICANCE TESTS BETWEEN MEAN INTERCEPTS}

One might not expect a bias in comparisons between average intercepts from two or more groups provided that the same stimulus measures were analyzed in each set. Unfortunately, the choice of scale for measuring the stimuli introduces problems with the power of significance tests. At times, comparisons of intercepts have been made to evaluate the stability of the units of subjects' responses with respect to changes in the experimental procedure or over different occasions for the same experimental procedure. Findings of nonsignificant differences between intercepts and between exponents have been taken as evidence of stable results. Obviously, the question of statistical power is particularly important for drawing such conclusions.

Assume that two sets of data are drawn from populations for which the average exponents are equal, but the scale units for subjects' responses differ between populations in such a way that the geometric mean response in one population, say the first, is greater than the geometric mean in the second population. Hence, the expected values of means of logarithmically transformed data are ordered

$$
E\left(\bar{Y}_{1}\right)>E\left(\bar{Y}_{2}\right),
$$

where $\bar{Y}_{1}$ and $\bar{Y}_{2}$ denote the means of samples from the two populations. To help simplify the expressions, the case is considered for tests using a normal deviate and 
for $r_{\bar{Y} b}=.0$. For this case, the test statistic for differences between intercepts is

$$
\begin{aligned}
z & =\frac{\bar{a}_{1}^{\prime}-\bar{a}_{2}^{\prime}}{\left(\frac{\sigma_{a_{1}^{\prime}}^{2}}{n_{1}}+\frac{\sigma_{a_{2}^{\prime}}^{2}}{n_{2}}\right)^{1 / 2}} \\
& =\frac{\left(\bar{Y}_{1}-\bar{Y}_{2}\right)-\bar{X}^{\prime}\left(\bar{b}_{1}-\bar{b}_{2}\right)}{\left[\frac{\sigma_{\bar{Y}_{1}}^{2}}{n_{1}}+\frac{\sigma_{\bar{Y}_{2}}^{2}}{n_{2}}+\bar{X}^{\prime 2}\left(\frac{\sigma_{b_{1}}^{2}}{n_{1}}+\frac{\sigma_{b_{2}}^{2}}{n_{2}}\right)\right]^{1 / 2}} .
\end{aligned}
$$

According to Equation 17, an increase in the absolute value of $\bar{X}^{\prime}$ increases the denominator term while adding to the numerator a random component with an expectation of zero. That is, an increase in $\bar{X}^{\prime}$ produces an increase in the standard error of the difference, but the expected difference between intercepts remains constant. The result is a decrease in the expected value of $z$ and, consequently, a decrease in power.

The influence of the stimulus measure on power is illustrated by a reanalysis of the Rule and Markley (1971) data from magnitude estimation of area. The data were randomly partitioned into two sets of data from 24 subjects each. The responses from Group 1 were increased by multiplying each response by 1.5 , thereby artificially increasing the size of subjects' responses in Group 1 relative to Group 2. The resulting geometric means for the two groups are 14.97 and 10.12 , which reflects the inflation of measures in the first group. The exponents from fitting power functions to each subject's data are unaffected by a change of unit of the responses. The mean exponents for the two groups are 0.74 and 0.71 .

When area is measured in square centimeters, the mean intercepts are -0.11 and -0.22 for Groups 1 and 2, respectively. The difference between mean intercepts was subjected to a $t$ test, which yielded a nonsignificant outcome of $t(46)=1.53, p>.05$. Another analysis of the data with area measured in square inches yielded mean intercepts of 0.49 and 0.35 , whose difference is significant $[t(46)=3.15, p<.01]$. The difference in the two outcomes, one significant and the other not, stems from the difference in the geometric means of the stimulus measures, $8.45 \mathrm{in}^{2}$ and $54.5 \mathrm{~cm}^{2}$. The lesser value for square inches reduced the standard error of a difference, and consequently increased $t$. When the stimuli are measured in square millimeters, with a geometric mean of $5,450 \mathrm{~mm}^{2}$, the test statistic is smaller still $[t(46)=0.29]$. Clearly, the power of the test can be dramatically affected by the arbitrary choice of scale for the measurement of the stimuli.

\section{RECOMMENDATIONS AND CONCLUDING COMMENTS}

The developments above indicate that the intercept is not an appropriate measure for analyses of subject differences or for the evaluation of stability of subjects' re- sponses. Instead, the results point to the geometric mean of a subject's estimates or its logarithm as the preferred measure of the unit of a subject's judgments. Shifts in the unit have a direct and predictable effect on these measures that is independent of the arbitrary choice of scale for measuring the stimuli. With respect to the question of a relation between the unit of response and the exponent, the correlation $r_{\bar{Y} b}$ for the data from magnitude estimation of area is .05 , which indicates little relationship. Such a result would seem consistent with expectation for data from magnitude estimation with a prescribed modulus, for which little variability is expected among units for different subjects. (However, a reanalysis of the Rule, 1969, data for area judgments for which no modulus was specified yielded a similar correlation between $\bar{Y}$ and the exponent of only .02.)

The geometric mean or its logarithm is also to be preferred for evaluating experimental effects such as converging power functions. When a set of power functions converges to a point above the range of the data, the logarithm of the geometric mean response to a common set of stimuli has a perfect negative correlation with the exponent. This relation is not influenced by the arbitrary choice of measurement scale for the stimuli as is the correlation between intercepts and exponents. Consequently, the size of $r_{\bar{Y} b}$ provides a direct index of how well convergence to a single point describes the data. When convergence is perfect, the correlation is -1.0 . The coordinates of the point of convergence can be estimated from the slope and intercept of the linear relation between the logarithm of the geometric means and the exponents as well. ${ }^{2}$

With respect to significance tests between sets of data, comparisons between $\bar{Y}_{1}$ and $\bar{Y}_{2}$ provide more powerful tests than do those between intercepts. For the example data in which one set was artificially inflated, the test statistic for the difference $\bar{Y}_{1}-\bar{Y}_{2}$ is $t(46)=6.66, p<.01$. A highly significant result is to be expected when one set of values has been inflated by $50 \%$. The replacing of the intercept with $\bar{Y}_{i}$ in the analysis of the stability of data suggests also replacing the exponent $b_{i}$ with the standard deviation of the logarithm of a subject's responses. Because the exponent is an imperfect measure of the standard deviation, substituting the standard deviation in the analysis would likely reduce error introduced by intermediate analyses.

With respect to the coefficient as an indication of the size of responses, it would seem that this is the case only when the geometric mean of the stimuli is one and, hence, its logarithm $\bar{X}^{\prime}$ is zero. This is the case for which the coefficient is equal to the geometric mean of the responses.

\section{REFERENCES}

Algom, D., Marks, L. E., \& Wiesenfeld, D. (1991). Tapping the social psychology of psychophysical experiments: Mode of responding does not alter statistical properties of magnitude estimates. Bulletin of the Psychonomic Society, 29, 226-228. 
BAird, J. C., Kreindler, M., \& Jones, K. (1971). Generation of multiple ratio scales with a fixed stimulus attribute. Perception \& Psychophysics, 9, 399-403.

BorG, G. A. V., Marks, L. E. (1983). Twelve meanings of the measure constant in psychophysical power functions. Bulletin of the Psychonomic Society, 21, 73-75.

BUtLer, D. L., \& OVershiner, C. (1983). The role of mental computations in judgments of area and volume. Perception \& Psychophysics, 34, 593-598.

Cross, D. V., \& Rotkin, L. (1975). The relation between size and apparent heaviness. Perception \& Psychophysics, 18, 79-87.

ENGEN, T. (1971). Psychophysics: II. Scaling methods. In J. W. Kling \& L. A. Riggs (Eds.), Woodworth \& Schlosberg's Experimental Psychology (3rd ed., pp. 47-86). New York: Holt, Rinehart \& Winston.

Ferguson, R. P., Martin, P. (1983). Long-term temporal estimation in humans. Perception \& Psychophysics, 33, 585-592.

Gescheider, G. A. (1988). Psychophysical scaling. Annual Review of Psychology, 39, 169-200.

Gescheider, G. A., \&ughson, B. A. (1991). Stimulus context and absolute magnitude estimation: A study of individual differences. Perception \& Psychophysics, 50, 45-57.

KowAL, K. H. (1987). Apparent duration and numerosity as afunction of melodic familiarity. Perception \& Psychophysics, 42, 122-131.

RoBINSON, G. H. (1992). Single-parameter power law psychophysics of auditory numerosity and the psychological moment hypothesis. Perception \& Psychophysics, 51, 363-378.

RuLE, S. J. (1969). Subject difference in exponents from circle size, numerousness, and line length. Psychonomic Science, 15, 284-285.

RULE, S. J., \& CURTIS, D. W. (1977). The influence of the interaction of weight and volume on subjective heaviness. Perception \& Psychophysics, 22, 159-164.

RUle, S. J., \& MarkLEY, R. P. (1971). Subject differences in crossmodality matching. Perception \& Psychophysics, 9, 115-117.

STEVENS, J. C. (1974). Families of converging power functions in psychophysics. In H. R. Moskowitz, B. Scharf, \& J. C. Stevens (Eds.),
Sensation and measurement: Papers in honor of S. S. Stevens (pp. 157165). Dordrecht: Reidel.

Stevens, J. C., \& Rubin, L. L. (1970). Psychophysical scales of apparent heaviness and the size-weight illusion. Perception \& Psychophysics, 8, 225-230.

\section{NOTES}

1. When $r_{\bar{Y} b}$ is nonzero, the equations for $r_{a b}$ and $r_{a} \bar{Y}$ are:

$$
r_{a b}=\frac{r_{\bar{Y} b} \sigma_{\bar{Y}}-\bar{X} \sigma_{b}}{\left(\sigma_{\bar{Y}}^{2}+\bar{X}^{2} \sigma_{b}^{2}-2 \bar{X} r_{\bar{Y} b} \sigma_{\bar{Y}} \sigma_{b}\right)^{1 / 2}}
$$

and

$$
r_{a} \bar{Y}=\frac{\sigma_{\bar{Y}}-\bar{X} r_{\bar{Y} b} \sigma_{b}}{\left(\sigma_{\bar{Y}}^{2}+\bar{X}^{2} \sigma_{b}^{2}-2 \bar{X} r_{\bar{Y} b} \sigma_{b} \sigma_{\bar{Y}}\right)^{1 / 2}} .
$$

2. A solution for the coordinates of the convergence point on the logarithmic scale, denoted by $X_{c}$ and $Y_{c}$, can be obtained from the slope and intercept of the linear relation:

$$
\bar{Y}_{i}=g+h b_{i},
$$

where

$$
g=Y_{c}
$$

and

$$
h=\bar{X}-X_{c} .
$$

Hence, $Y_{c}=g$ and $X_{c}=\bar{X}-h$. Recall that $h$ is negative.

(Manuscript received August 24, 1992; revision accepted for publication March 23, 1993.) 